\title{
Rotavirus hijacks host gluconeogenesis-related signaling pathway to promote self-replication in Caco- 2 cells
}

\section{Lijun Song ( $\square$ songlijun6981@126.com )}

Guangdong Medical University

\section{Peicheng Zhong}

Guangdong Medical University

\section{Mengyue Gao \\ Guangdong Medical University}

\section{Qian Lan}

Guangdong Medical University

Xuemei Zhu

Guangdong Medical University

Jiabo Chen

Guangdong Medical University

\section{Yang Chen}

Guangdong Medical University

\section{Yujing Zhou}

Guangdong Medical University

\section{Yuxuan Feng}

Guangdong Medical University

\section{Guiqin Dai}

Guangdong Medical University

\section{Feng Li}

Guangdong Medical University

\section{Wenchang Zhao}

Guangdong Medical University

\section{Original Article}

Keywords: Rotavirus (RV), Gluconeogenesis, Caco-2 cells

Posted Date: January 29th, 2021

DOl: https://doi.org/10.21203/rs.3.rs-162787/v1 
License: (c) (i) This work is licensed under a Creative Commons Attribution 4.0 International License. Read Full License 


\section{Abstract}

Viruses have evolved mechanisms to usurp the host's metabolic resources for their own biosynthesis and replication, but host's glucose metabolism change after rotavirus (RV) stress remains unclear. The metabolic profile and differential gluconeogenesis analysis was performed by gas chromatography-mass spectroscopy on RV-infected cells.47 potential differential metabolites were identified to verify glycolytic/gluconeogenesis pathway after RV infection. Glucose consumption and key enzymes of gluconeogenesis (glucose 6-phosphatase (G-6-pase) and phosphoenolpyruvate carboxylase (PEPCK)) were further examined by glucose oxidase-peroxidase method and enzyme linked immunosorbent assay. We found the impairment of glucose consumption, G-6-pase and PEPCK activities which promote gluconeogenesis, were tested in RV infected Caco-2 cells. By Western blot analysis, further studies detected the downregulation expression level of SIK2, which was affected by its upstream proteins of downregulated PDK1, AKT and upregulation p-JNK, and as a consequence influenced the function of gluconeogenesis. In short, RV infection altered the glucose consumption in infectious Caco-2 cells and activated the host cellular gluconeogenesis, which was hijacked through p-JNK-PDK1-AKT-SIK2 signaling pathway for its proliferation and replication with accelerating non-sugar substances conversion into glucose.

\section{Introduction}

Viruses as obligate has evolved mechanisms that target the host cellular energy and macromolecule synthesis as part of their replication cycle, creating specific microenvironments required for various stages of their lives cycles(1). However, the detailed mechanism by which viruses alter energy metabolism, especially glucose metabolism, remains completely unclear.

Recently research has focussed on examining how virus infection alters various glucose metabolism profiles of host cells, including glucose transport, insulin signaling pathways and glycolysis. The glucose transporters adjustment effects of virus infection have been investigated for several viruses, including human T-cell leukemia virus(2), white spot syndrome virus(3), Kaposi's sarcoma-associated herpesvirus(4), human cytomegalovirus(5) and hepatitis $C$ virus(6), which affected the glucose supply. Various viruses including poliocirus(7), influenza A(8), human T-cell leukemia virus(9), HSVT-1(10), HIV1(11), Mayaro virus(12) and HCMV(13), have been shown to activate Warburg effect under aerobic conditions, which enhanced glucose uptake and glycolytic activities. Furthermore, hepatitis $\mathrm{C}$ virus interfered with key enzymes in the gluconeogenesis and pentose phosphate pathways, and even disrupted the function of islet cell(14).

Rotaviruses (RVs), enteropathogenic viruses that infect over 114 million children worldwide(15), invades enterocyte to stimulate enteric nervous system and chlorinated secretions depending on calcium ions $(16,17)$. Recent research showed RV infection downregulated sodium-glucose transport 1 expression and decreased the activity of brush border disaccharidases(18). Normally, RV relies on host cellular metabolism for the energy and macromolecule synthesis required for their replication. And positive 
clinical evidence indicates that type 1 diabetes in children partly resulted from RV infection(19). Therefore, deepening the understanding of the glucose metabolic alterations required for the RV replication becomes an urgent problem to be solved.

In this study, abnormal glucose consumption was found in RV-infected Caco-2 cells, which indicated that $\mathrm{RV}$ infection indeed changed the glucose metabolism. On basis of this, metabolomics was performed to screen out the differential metabolites and the gluconeogenesis pathway was revealed by bioinformation. The elevated expression of its associated G-6-pase and PEPCK were also verified after RV infection. In addition, p-JNK-PDK1-AKT-SIK2 signaling pathway was activated leading to the RV-induced upregulation of host cellular gluconeogenesis. These results indicate that RV infection facilitates the host cellular gluconeogenesis approach for its biosynthesis.

\section{Material And Methods}

\subsection{Reagents}

Caco-2 cell line was from cell bank of Wuhan University (Wuhan, China). The MA104 cell line was from the cell bank of Sun Yat-Sen University (Guangzhou, China). The RV Wa strain (G1P[8]) was was from the immunology institute of the Third Military Medical University (Chongqing, China). The glucose assay kit was obtained from Shanghai Rong Sheng Biotechnology Co., LTD (Shanghai, China). The glucose 6phosphatase and phosphoenolpyruvate carboxylase assay kits were from Solarbio (Beijing, China). AntiJNK, anti-AKT, anti-SIK2 and anti-PDK1 were obtained from Proteintech (Wuhan, China). Anti-beta-actin, anti-rabbit IgG were obtained from Cell Signaling Technology (Boston, USA).

\subsection{Cell culture}

Caco-2 and MA104 cells were cultured with high-sugar DMEM medium containing $1 \%$ anti-penicillin, anti-streptomycin and $10 \% \mathrm{FBS}$, which were placed in the cell incubator at constant $37^{\circ} \mathrm{C}$ and $5 \% \mathrm{CO}_{2}$ for conventional culture. When the cells grew to about $85 \%$ $90 \%$, original medium was disposed. And an appropriate amount of trypsin containing $0.25 \%$ EDTA was added and incubated for 2 min $7 \mathrm{~min}$. Then fresh culture medium was added to stop digestion. Next, the mixed suspension of cells was centrifuged at $900 \mathrm{rpm} / \mathrm{min}$ for $3 \mathrm{~min}$, and the supernatant was removed. Then $4 \mathrm{ml} \mathrm{DMEM}$ culture medium was added for cell suspension, which was divided into new culture bottles according to a certain proportion. Finally, $7 \mathrm{ml}$ fresh culture medium was added to continue the routine culture.

\subsection{Cultivation and amplification of RV on MA104 cells}


Virus were removed from $-80^{\circ} \mathrm{C}$ and dissolve at $4^{\circ} \mathrm{C} .10 \mu \mathrm{g} / \mathrm{ml}$ trypsin without EDTA was added and incubated at $37^{\circ} \mathrm{C}$ for $30 \mathrm{~min}$. MA104 cells, which had grown to a monolayer, were added with incubated $\mathrm{RV}$ venom. MA104 cells with RV were routinely cultured at $37^{\circ} \mathrm{C}$ and $5 \% \mathrm{CO}_{2}$. After the cytopathic effect of MA104 cells were observed under an inverted microscope, the cell culture bottle was taken out and put at $-20^{\circ} \mathrm{C}$ for $12 \mathrm{~h}$. Next, the cell was placed at $4{ }^{\circ} \mathrm{C}$ for natural melting, and the above operations were repeated for 3 times. The cell freeze-thaw solution was collected in the centrifuge tube, and centrifuged at $12000 \mathrm{~g}$ for $30 \mathrm{~min}$. Finally, the supernatant RV venom was collected and stored at $-80^{\circ} \mathrm{C}$. For the titer determination of $R V$, the incubated $R V$ venom was first diluted into a series of concentrations, including $10^{-1}, 10^{-2}, 10^{-3}, 10^{-4}, 10^{-5}$, and $10^{-6}$, in DMEM culture solution without fetal bovine serum, and then applied to MA104 cells at $100 \mu \mathrm{l} /$ well. The pathological changes of MA104 cells after RV infection were observed at different times. When a CPE was no longer present in the 96-well plates of RV venom with the lowest dilution, the number of wells with a CPE was recorded for each dilution. The median tissue culture infective dose (TCID50) of the virus was calculated using the Reed and Muench method.

\subsection{Preparation and grouping of Caco-2 cells infected with RV}

The RV venom at a virus titer of $10^{5} \mathrm{TCID} 50 / \mathrm{ml}$ (the virus reacted with $10 \mu \mathrm{g} / \mathrm{ml}$ trypsin for $30 \mathrm{~min}$ ) was added to the $100 \mathrm{~mm} \times 100 \mathrm{~mm}$ culture dish where Caco- 2 cells grew to a monolayer. At the same time, the uninfected Caco- 2 cells was set and the equal volume DMEM culture medium was added. After incubated at $37^{\circ} \mathrm{C}$ with $5 \% \mathrm{CO}_{2}$ for $2 \mathrm{~h}$, the cells' RV venom was replaced with DMEM culture solution. After 48 hours of continuous culture, the RV-infected Caco-2 cells model was successfully constructed.

\subsection{GC-MS analysis of cellular metabolites}

Preparation of samples: the RV-infected and uninfected Caco-2 cells were scraped off by adding $4^{\circ} \mathrm{C}$ precooled methanol and transferred into the centrifuge tube. The mixture was centrifuged at $4{ }^{\circ} \mathrm{C}$ and $1.4 \mathrm{x}$ $10^{4} \mathrm{~g}$ for $15 \mathrm{~min}$ and take $500 \mu \mathrm{l}$ supernatant with $10 \mu \mathrm{l}$ internal standard (50 $\mu \mathrm{g} / \mathrm{ml} \mathrm{L}$-norvaline). After dried under nitrogen, $40 \mu \mathrm{l}$ methylamine hydrochloride pyridine was added and the supernatant was incubated at $37^{\circ} \mathrm{C}$ for 90 min. Finally, added with 40 LBSTFA (including $1 \%$ trimethyl chlorosilane), it vortexed for 30 seconds and derived at $70^{\circ} \mathrm{C}$ for 60 minutes. Samples derived from trimethylsilane were obtained for GC-MS analysis.

GC-MS conditions: Agilent gas chromatogram mass spectrometer (7890A/5975C) and MACHEREYNAGEL OPTIMA®5 MSAccent fused silicon capillary column (30 m x $0.25 \mathrm{~mm} \times 0.25 \mu \mathrm{m})$ were used to 
conduct metabolomics tests on derived samples. The obtained data files were imported into SIMCA software (version 14.1) of UmetricsAB for multidimensional statistical analysis such as principal component analysis (PCA), partial least-square-discriminant analysis (PLS-DA) and orthogonal filtering partial least-square-discriminant analysis (OPLS-DA). The model quality is described by the $R^{2} X$ or $R^{2} Y$ and $Q^{2}$ values. $R^{2} X(P C A)$ or $R^{2} Y$ (PLS-DA and OPLS-DA) is defined as the proportion of variance in the data explained by the models and indicates the goodness of fit. $\mathrm{Q}^{2}$ is defined as the proportion of variance in the data predictable by the model and indicates the predictability of current model, calculated by cross-validation procedure. Generally, their value is greater than 0.5 , which means that the model quality is better.

Metabolite structure identification method: The AMDIS software was used for GC-MS deconvolution analysis of original data automatically, which was matched self-built standard database (including retention time and mass spectrum), Golm metabolome database and Agilent Fiehn GC/MS metabonomics RTL database.

\subsection{Analysis of gluconeogenesismetabolic enzymes}

The supernatant of cell culture with RV infection was disposed, and then cells were scraped and collected in the centrifuge tube. The cells were centrifuged at $10000 \mathrm{~g}$ for $10 \mathrm{~min}$, and the precipitation was preserved. $1 \mathrm{ml}$ of working solution was added into the precipitate, which crushed by ultrasonic. According to the enzyme assay kit instructions, G-6-Pase and PEPCK activity were detected.

\subsection{Analysis of glucose consumption}

The glucose assay kit (glucose oxidase-peroxidase method) was used to detect the glucose content in the supernatant of cell culture in Caco-2 cells infected by RV, in order to determine the glucose consumption of Caco- 2 cells after RV treatment.

\subsection{Western blot}

After Caco- 2 cells were grown in a $6 \mathrm{~cm}$ petri dish and the experimental interventions were conducted, Radio immunoprecipitation assay lysis buffer (containing 1\% PMSF) was added for cleavage. The protein samples were then added to the $5 x$ SDS loading buffer and denatured at $95^{\circ} \mathrm{C}$ for $5 \mathrm{~min}$. The denatured protein samples were separated by SDS-PAGE. It was transferred to PVDF membrane through 
wet transfer and incubated with corresponding antibodies. The dilution multiples of antibodies were as follows: JNK (1:500), PDK1 (1:500), AkT (1:2000), SIK2 (1:1000), and beta-actin (1:1000). Finally, protein expression levels were analyzed by Image $\mathrm{J}$ software, with beta-actin as internal reference.

\subsection{Statistical approach}

SPSS 13.0 statistical software was used for data analysis. T test was used to compare the two groups of control experiments, and One-Way ANOVA method was used to compare the mean of multiple samples. The experimental results were expressed as mean \pm standard deviation $(x \pm s)$, and $P<0.05$ was statistically significant.

\section{Results}

\subsection{Impairment of glucose consumption in RV-infected Caco-2 cells}

The level of glucose consumption is used to indicate the ability of cells to consume extracellular glucose. The results showed that the glucose consumption of RV-infected Caco-2 cells in the medium decreased significantly $(P<0.05$, v.s. uninfected Caco-2 cells, Fig. $1 \mathrm{~A})$.

\subsection{RV infection altered the gluconeogenesis metabolism in Caco-2 cells}

In order to determine changes in specific glucose metabolism pathways in Caco-2 cells after RV infection, high-throughput GC-MS analysis was performed(20).

First, the data were processed to analyze the metabolic profile of the RV-infected and uninfected Caco-2 cells. As shown in the total ion current (TIC) chromatogram (Fig.1B), metabolites in RV-infected and uninfected Caco-2 cells were well separated. The retention time of the chromatogram was mainly concentrated at 5-21 min, and there were significant differences in the retention time and peak area of the metabolites.

Principal component analysis ( $\left.P C A, R^{2} X=0.629\right)(21)$ and Partial least-squares discriminant analysis (PLS$D A, R^{2} X=0.578, R^{2} Y=0.999, Q^{2}=0.963$ ) were performed on the metabolic profile data to detect the change of metabolic profile between the RV-infected and uninfected Caco-2 cells (Fig.1C,D). The both diagrams showed it could be separated and had obvious clustering characteristics, revealing significant metabolic 
differences between the samples of RV-infected and uninfected Caco-2 cells. It is also seen that the model was non-overfitting with Permutation test (Fig.1E, the criterion of non-overfitting models is that the value of the cut point between the Q2 slash and the $\mathrm{Y}$-axis is less than 0 , or the point to the right of Q2 is less than all the points to the left).

Next, the markers were identified. According to the result of orthogonal partial least-squares discriminant analysis (OPLS-DA, $R^{2} X=0.578, R^{2} Y=0.999, Q^{2}=0.973$, Fig. $1 F$ ), it was found that it could be divided into two parts between the samples of RV-infected and uninfected Caco-2 cells. It indicated that the difference between groups was much greater than the difference within groups. Thus, the samples of RV-infected and uninfected Caco-2 cells could be effectively distinguished. The variable projection (VIP) importance of the first principal component from OPLS-DA model (threshold $>1$ ) and the p-value of the onedimensional test (threshold $<0.05$ ) were used as the criteria for judging the differential expression of metabolites. The qualitative method of differential metabolites was to search the self-established standard material database, including chromatographic retention time and mass spectrometry. As a result, a total of 47 differentiated substances were identified, of which 20 decreased and 27 increased.

To further characterize the correlations among metabolites of differences, we performed Pearson Correlation analysis (Fig.1G) on the quantitative information of these metabolites. Compared with the uninfected Caco-2 cells, dihydroxyacetone phosphate was significantly decreased in the RV-infected Caco-2 cells, while glucose 6-phosphate, fructose 6-phosphate, fumaric acid, succinic acid and malic acid were significantly increased. These metabolites are closely related to the gluconeogenesis of host cells. Dihydroxyacetone phosphate is the key product of gluconeogenesis. It could convert into glucose 6phosphate, which is not only the intermediate material of the gluconeogenesis, but also the intersection point between pentose phosphate pathway and glycolysis. In addition, fumaric acid, succinic acid and malic acid are associated with the TCA cycle, which are intermediates of gluconeogenesis and glycolysis. It indicated that RV infection makes use of the sugar in the cell or uses other substances to generate sugars or lipids for survival(22-24).

Finally, online software MetaboAnalyst (version 4.0) was used to conduct pathway analysis on the differential metabolites (Fig.1H). Gluconeogenesis pathway was worthy of study with low P values. Gluconeogenesis is a way of glucose synthesis from non-carbohydrate precursors, which is essentially a reversal of glycolysis. Based on the results above, we chose the gluconeogenesis pathway as the study of the mechanism of RV infection in Caco-2 cells.

\subsection{The elevation of G-6-pase and PEPCK activity in RV infected Caco- 2 cells}


To further verify the effect of RV infection on the gluconeogenesis pathway in host cells, we analyzed the key enzyme activity related the gluconeogenesis. G-6-pase is a phosphatase that hydrolyzes phosphate compounds. It regulates the amount of glucose released into the blood by hydrolyzing glucose6phosphate. PEPCK is an enzyme that catalyzes the conversion of oxaloacetic acid into phosphoenolpyruvate. Both enzymes are rate-limiting in the gluconeogenesis pathway, whose activity or transcription determines the efficiency of gluconeogenesis. The results showed that the activity of G-6Pase and PEPCK in the RV-infected and Caco-2 cells was significantly facilitated compared with that in the uninfected Caco-2 cells. It indicated that the gluconeogenesis activity of Caco-2 cells accelerated after RV infection (Fig.2A).

\subsection{RV facilitated the activity of G-6-pase and PEPCK via p-JNK- PDK1- AKT- SIK2 signaling pathway}

Given the activation of G-6-Pase and PEPCK by RV infection, we assumed that the gluconeogenesis promotion was related to cyclic adenosine phosphate reaction element binding protein "CREB"(25), which initiate gluconeogenesis after combined withtarget of rapamycin complex 2 (TORC2) $(26,27)$. It's corresponding to the result that salt-inducible kinase 2 (SIK2) expression in RV-infected Caco-2 cells was down-regulated with statistical difference (Fig 2b,c), which resulted in less phosphorylated TORC2 and formation of CREB-TORC2 complex(28). In addition, SIK2 activation is enhanced by phosphorylation at $\mathrm{Ser}^{358}$, a process catalyzed by protein kinase B (AKT)(29) that was down-regulated in RV-infected Caco-2 cells (Fig.2B,C), thus reducing the activation of SIK2, which was consistent with the above result. Further, in view of PI3K/AKT signal pathway, we found that the expression of 3-phosphoinositide-dependent protein kinase-1 (PDK1) in Caco-2 cells was inhibited after RV infection (Fig.2B,C), which down-regulated the expression of AKT. Furthermore, the JNK signaling pathway is activated in the case of RV infection stress(30) based on the result that the expression of phosphorylated JNK (p-JNK) in RV-infected Caco-2 cells was up-regulated, which also could inhibit the PDK1 level referring to the insulin resistance signaling pathway (Fig.2B,C). As was demonstrated above,RV infection hijacked host gluconeogenesis-related pJNK-PDK1-AKT-SIK2 signaling pathway to improve gluconeogenesis activity (Fig.2D).

\section{Discussion}

$\mathrm{RV}$ is the leading cause of diarrhea in infants, which leads to lactose intolerance and down-regulation of sodium-glucose transport 1 in intestine(31-33). Beyond this, RV could also be found in the different sites of CSF, heart, testes, kidneys, liver, lung, and bladder(34), which cause seizures, cerebellitis, pancreatitis and antigenemia(35-37). There is also research suggesting that $\mathrm{RV}$ infection has been claimed as a triggering factor for type I diabetes mellitusin children, which selectively associated islet autoantibody 
markers and destructed the insulin-producing pancreatic $\beta$ cells $(38,39)$. Therefore, $R V$ infection is closely related to energy metabolism, especially glucose metabolism.

In this study, we found that RV infection can reduce glucose in host cells, and gluconeogenesis pathway related to glucose uptake was screened out from the metabolomics results, which plays an essential role in maintaining the normal blood glucose level. It was further found that RV infection indeed enhanced the activity of gluconeogenesis enzymes such as G-6-Pase and PEPCK. These results suggested that RV not only alterd the uptake of glucose by host cells, but also hijacked the gluconeogenesis pathway, accelerating the rapid intracellular conversion of non-sugar substances into glucose for its own use. On the one hand, the decreased glucose uptake of host cells would lead to the decreased function of intestinal epithelial cells, and even apoptosis or death(40). Meanwhile, intracellular viruses hijack the cell's gluconeogenesis pathway, thus interfering with and utilizing the normal intracellular energy metabolism. At a deeper level, gluconeogenesis was regulated by the CBP-CREB-TORC2 complex and SIK2, while RV infection could change the expression of SIK2. Furthermore, the upstream regulatory proteins of SIK2 include AKT, PDK1 and p-JNK. These results provide important experimental basis for the discovery of the key molecular and glucose regulatory targets of RV infection, which was also the experimental basis for the treatment of RV infection through metabolic intervention.

\section{Declarations}

\section{Author contribution}

Conceptualization:Wenchang Zhao. Methodology: Lijun Song,Peicheng Zhong,Mengyue Gao,Qian Lan.Formal analysis and investigation: Lijun Song,Peicheng Zhong,Xuemei Zhu,Jiabo Chen,Yang Chen. Writing-original draft preparation:Lijun Song,Peicheng Zhong,Yujing Zhou,Yuxuan Feng,Guiqin Dai,Feng Li.Writing-review and editing: Lijun Song,Wenchang Zhao,Peicheng Zhong, Funding acquisition:Wenchang Zhao.Resources:Wenchang Zhao.Supervision:Wenchang Zhao,Lijun Song.All authors provided critical feeedback and and helped shape the research,analysis and manuscript.

\section{Ethics declarations}

Conflict of interest

We declare that we have no conflicts of interest to this work. Lijun Song and Peicheng Zhong are co-first authors.

Research involving human and animal rights

This article does not contain any studies with human participants or animals performed by any of the authors.

Consent for publication 
Authors agree to data publication and informed about the Publisher rules and terms.

\section{Acknowledgments}

This study was funded by National Natural Science Foundation of China (No. 81973548 and No. 81473401), Key Project of Social Science and Technology Development of Dongguan (NO. 20185071521658) and 2018 Guangdong Graduate Education Innovation Program (NO. 2019XSLT29).

\section{References}

1. Wu SW, Fu XZ, Lin Q, et al. Research Progress in Virus Infection Altering Cellular Glucose Metabolism. Chinese Journal of Virology. 2016; 32:800-809.

2. Jin Q, Alkhatib B, Cornetta K, et al. Alternate receptor usage of neuropilin-1 and glucose transporter protein 1 by the human T cell leukemia virus type 1. VIROLOGY. 2010; 396:203-212.

3. Huang HT, Chan HL, Shih TY, et al. A study of the role of glucose transporter 1 (Glut1) in white spot syndrome virus (WSSV) infection. Fish Shellfish Immunol. 2015; 46:305-314.

4. Dalla PA, Pinato DJ, Bracchi M, et al. Recent advances in HIV-associated Kaposi sarcoma. 2019; F1000Res 8.

5. Carvalho E, Schellhorn SE, Zabolotny JM, et al. GLUT4 overexpression or deficiency in adipocytes of transgenic mice alters the composition of GLUT4 vesicles and the subcellular localization of GLUT4 and insulin-responsive aminopeptidase. J BIOL CHEM. 2004; 279:21598-21605.

6. Sun LJ, Yu JW, Wan L, et al. Endocannabinoid system activation contributes to glucose metabolism disorders of hepatocytes and promotes hepatitis C virus replication. INT J INFECT DIS. 2014; 23:7581.

7. EAGLE H, HABEL $K$. The nutritional requirements for the propagation of poliomyelitis virus by the HeLa cell. J EXP MED. 1956; 104:271-287.

8. KLEMPERER H. Glucose breakdown in chick embryo cells infected with influenza virus. VIROLOGY. 1961; 13:68-77.

9. Manel N, Kim FJ, Kinet S, et al. The ubiquitous glucose transporter GLUT-1 is a receptor for HTLV. CELL. 2003; 115:449-459.

10. GIL FC. [Influence of various factors on the development of herpes simplex virus in HeLa cells]. Microbiol Esp. 1959; 12:297-323.

11. Tritel M, Resh MD. The late stage of human immunodeficiency virus type 1 assembly is an energydependent process. J VIROL. 2001; 75:5473-5481.

12. El-Bacha T, Menezes MM, Azevedo ESM, et al. Mayaro virus infection alters glucose metabolism in cultured cells through activation of the enzyme 6-phosphofructo 1-kinase. MOL CELL BIOCHEM. 2004; 266:191-198.

13. Landini MP. Early enhanced glucose uptake in human cytomegalovirus-infected cells. J GEN VIROL. 1984; 65 ( Pt 7):1229-1232. 
14. Li YP, Niu JQ. Relationship between chronic hepatitis $C$ virus infection and antiviral therapy and pancreatic $\beta$-cell function. Chinese Journal of Infectious Diseases. 2014; 32:187-189.

15. LeClair CE, Budh DP. Rotavirus. In: StatPearls [Internet]. Treasure Island (FL): StatPearls. Publishing: 2020 Jan-; Available at: <https://www.ncbi.nlm.nih.gov/books/NBK558951/>.

16. Lundgren $\mathrm{O}$, Peregrin AT, Persson $\mathrm{K}$, et al. Role of the enteric nervous system in the fluid and electrolyte secretion of rotavirus diarrhea. SCIENCE. 2020; 287:491-495.

17. Hagbom $M$, Istrate $C$, Engblom D, et al. Rotavirus stimulates release of serotonin (5-HT) from human enterochromaffin cells and activates brain structures involved in nausea and vomiting. PLOS PATHOG. 2011; 7:e1002115.

18. Lorrot M, Vasseur M. How do the rotavirus NSP4 and bacterial enterotoxins lead differently to diarrhea? VIROL J. 2007; 4:31.

19. van der Werf N, Kroese FG, Rozing J, et al. Viral infections as potential triggers of type 1 diabetes. Diabetes Metab Res Rev. 2007; 23:169-183.

20. Wang GJ, Cha WB, Hao HP, et al. Metabonomics and its Application Prospect in TCM Study. CHINESE JOURNAL OF NATURAL MEDICINES. 2008; (02):89-97.

21. van den Berg RA, Hoefsloot HC, Westerhuis JA, et al. Centering, scaling, and transformations: improving the biological information content of metabolomics data. BMC GENOMICS. 2006; 7:142.

22. Cheung W, Gill M, Esposito A, et al. Rotaviruses associate with cellular lipid droplet components to replicate in viroplasms, and compounds disrupting or blocking lipid droplets inhibit viroplasm formation and viral replication. J VIROL. 2010; 84:6782-6798.

23. Dickman KG, Hempson SJ, Anderson J, et al. Rotavirus alters paracellular permeability and energy metabolism in Caco-2 cells. Am J Physiol Gastrointest Liver Physiol. 2000; 279:G757-G766.

24. Gaunt ER, Cheung W, Richards JE, et al. Inhibition of rotavirus replication by downregulation of fatty acid synthesis. J GEN VIROL. 2013; 94:1310-1317.

25. Ge J, Zhang Y, Zheng ZZ, et al. Research Progress on CREB and the signal transduction pathways of its Phosphorylation. JOURNAL OF ANHUI AGRICULTURAL SCIENCES. 2010; 38:16769-16771.

26. Ravnskjaer K, Kester H, Liu Y, et al. Cooperative interactions between CBP and TORC2 confer selectivity to CREB target gene expression. EMBO J. 2007; 26:2880-2889.

27. Canettieri G, Koo SH, Berdeaux R, et al. Dual role of the coactivator TORC2 in modulating hepatic glucose output and insulin signaling. CELL METAB. 2005; 2:331-338.

28. Liu Y, Li J, Jin DQ, et al. Regulation of TORC-CREB Complex by Salt-inducible kinases: Implications in Hypertension and Diabetes Mellitus. PROGRESS IN BIOCHEMISTRY AND BIOPHYSICS. 2009; 36:274279.

29. Dentin R, Liu Y, Koo SH, et al. Insulin modulates gluconeogenesis by inhibition of the coactivator TORC2. NATURE. 2007; 449:366-369.

30. Solinas G, Becattini B. JNK at the crossroad of obesity, insulin resistance, and cell stress response. MOL METAB. 2017; 6:174-184. 
31. Chen XZ, Coady MJ, Jackson F, et al. Thermodynamic determination of the $\mathrm{Na}+$ : glucose coupling ratio for the human SGLT1 cotransporter. BIOPHYS J. 1995; 69:2405-2414.

32. Wright EM, Loo DD, Hirayama BA, et al. Surprising versatility of Na+-glucose cotransporters: SLC5. Physiology (Bethesda). 2004; 19:370-376.

33. Halaihel $\mathrm{N}$, Lievin $\mathrm{V}$, Alvarado $\mathrm{F}$, et al. Rotavirus infection impairs intestinal brush-border membrane $\mathrm{Na}(+)$-solute cotransport activities in young rabbits. Am J Physiol Gastrointest Liver Physiol. 2000; 279:G587-G596.

34. Alfajaro MM, Cho KO. Evidences and consequences of extra-intestinal spread of rotaviruses in humans and animals. Virusdisease. 2014; 25:186-194.

35. Paul SP, Candy DC. Extra-intestinal manifestation of rotavirus infection....Beyond the gut. INDIAN J PEDIATR. 2014; 81:111-113.

36. Jalilvand S, Marashi SM, Tafakhori A, et al. Extraintestinal Involvement of Rotavirus Infection in Children. ARCH IRAN MED. 2015; 18:604-605.

37. Blutt SE, Kirkwood CD, Parreno V, et al. Rotavirus antigenaemia and viraemia: a common event? LANCET. 2003; 362:1445-1449.

38. Honeyman MC, Coulson BS, Stone NL, et al. Association between rotavirus infection and pancreatic islet autoimmunity in children at risk of developing type 1 diabetes. DIABETES. 2000; 49:1319-1324.

39. Pane JA, Fleming FE, Graham KL, et al. Rotavirus acceleration of type 1 diabetes in non-obese diabetic mice depends on type I interferon signalling. Sci. 2016; Rep 6:29697.

40. Bhowmick R, Halder UC, Chattopadhyay S, et al. Rotaviral enterotoxin nonstructural protein 4 targets mitochondria for activation of apoptosis during infection. J BIOL CHEM. 2012; 287:35004-35020.

Figures
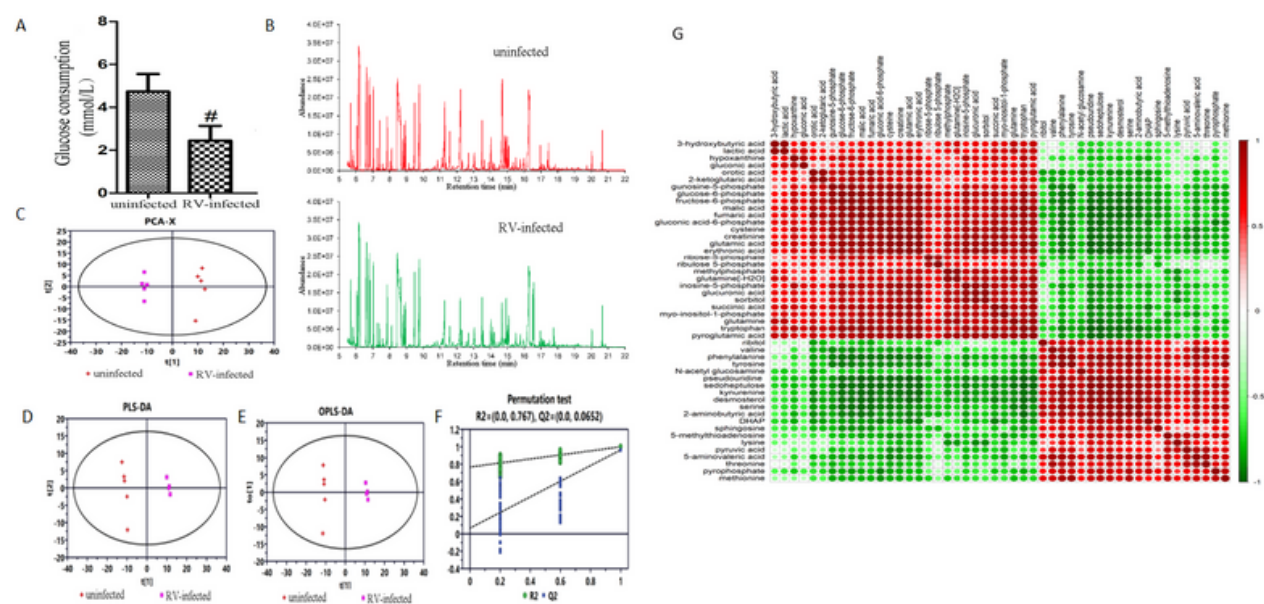

H

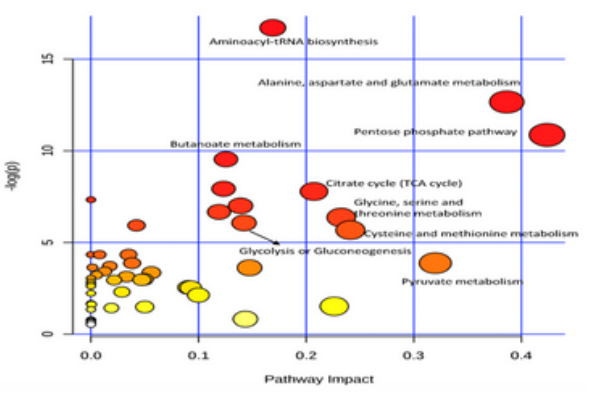

\section{Figure 1}

The changes in glucose consumption and gluconeogenesis pathway in Caco-2 cells after RV infection: A. Analysis of glucose consumption in Caco-2 cells after RV infection (\#P<0.05); $\mathrm{B}$. TIC chromatogram of 
RV-infected and uninfected Caco-2 cells collected by mass spectrometry full scan mode(mass/charge ratio $(\mathrm{m} / \mathrm{z}$ ) ranges from 50 to 600$)$; C. The metabolomics PCA of the RV-infected and uninfected Caco-2 cells (abscissa represents the first principal component, $\mathrm{PC1}, \mathrm{t}[1]$; the ordinate represents the second principal component, PC2, t[2].); D. The metabolomics PLS-DA of the RV-infected and uninfected Caco-2 cells; E. Permutation test diagram (Mainly used to characterize whether the model is overfitting. The method to judge whether the model is over-fitting is that the value of the Q2 diagonal line and the $\mathrm{Y}$-axis cutting point is less than 0 or the rightmost point of Q2 is less than all the points on the left); $F$. The metabolomics OPLS-DA of the RV-infected and uninfected Caco-2 cells; G. Pearson Correlation analysis diagram, each row and column represent a different metabolite. Red indicates a positive correlation and green indicates a negative correlation between different metabolites. The darker the color, the larger the square and the greater the correlation; $\mathrm{H}$. Pathway analysis diagram (The higher the $\mathrm{p}$ value is, the lower the possibility that metabolites in this pathway are related to classification and otherwise the opposite)

A

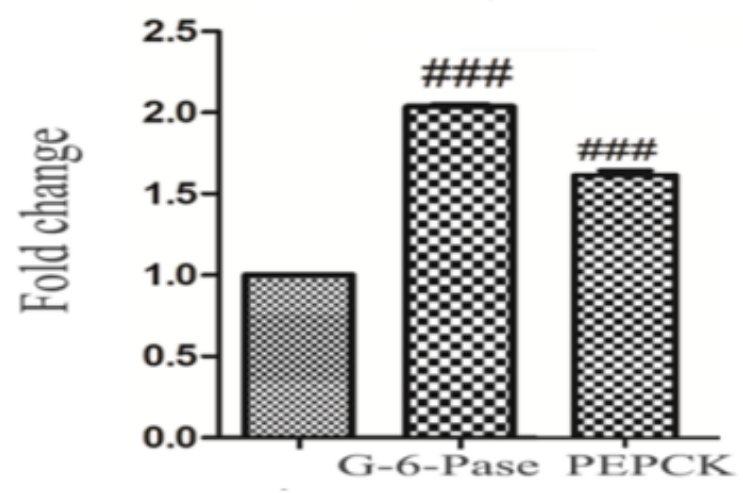

C

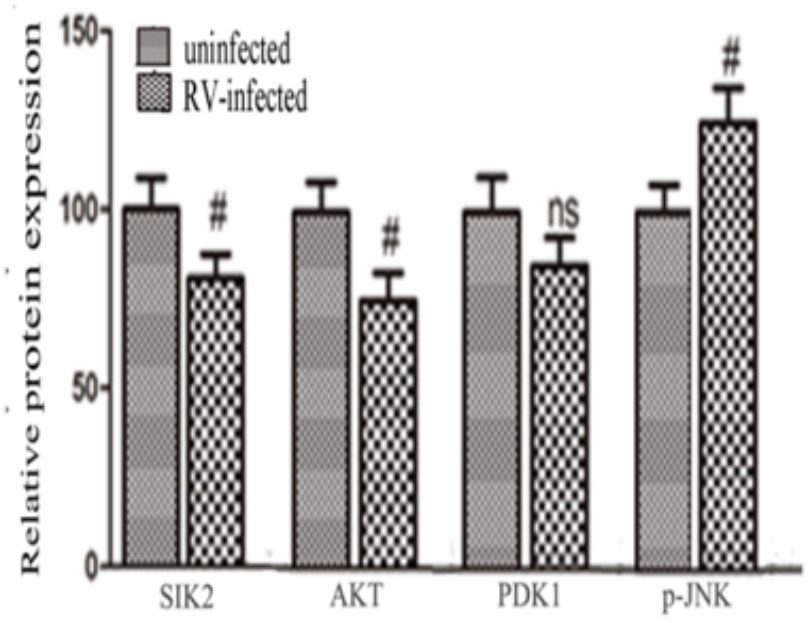

B

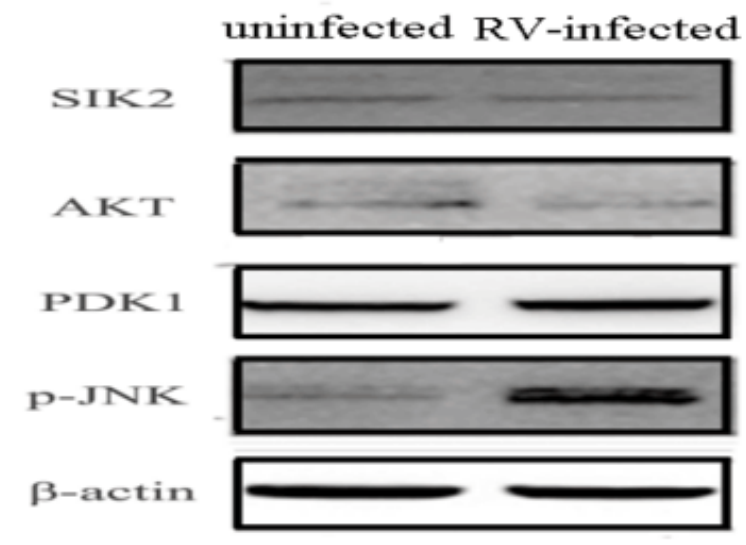

D

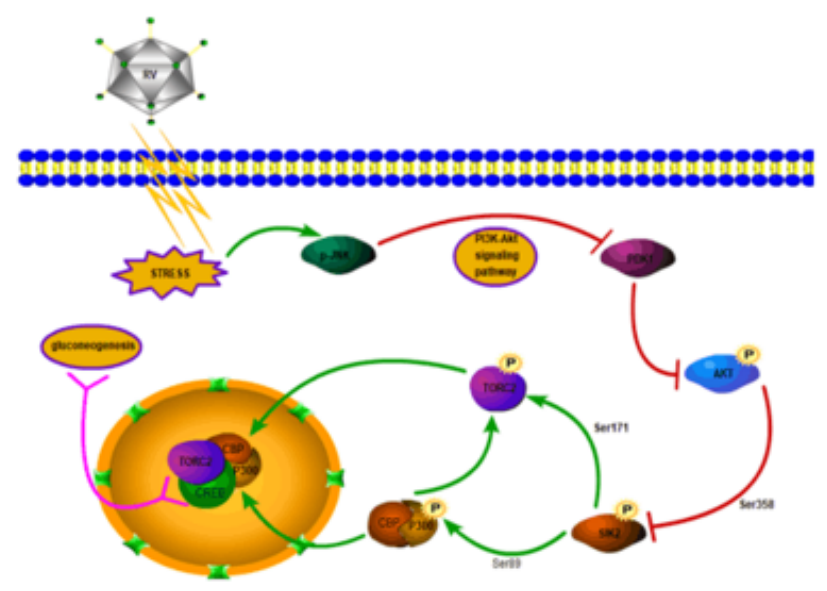

\section{Figure 2}

The changes in gluconeogenesis-related enzymes and signaling pathways in Caco- 2 cells after RV infection: A. The activity of G-6-Pase and PEPCK in Caco-2 cells after RV infection (\#\#\#P<0.001); B C. 
The expression level of SIK2, AKT, PDK1 and p-JNK protein in Caco-2 cells after RV infection (\#P < 0.05; $\mathrm{ns}$, not significant). D. The stimulation of cells by RV caused the overexpression of protein $\mathrm{p}-\mathrm{JNK}$, which downregulated the expression of protein PDK1 and AKT through the PI3K-AKT signaling pathway. And protein SIK2 can be activated by AKT phosphorylation, thereby promoting the entry of TORC2 and CBPP300 into the nucleus for CREB complex form and turn on gluconeogenesis. 